\title{
Etude et tenue de quelques matériaux à la cavitation
}

\author{
J.M. Roman, L. Finet \\ Sté Neyrpic, \\ 75, rue Général Mangin, B.P. 75, \\ 38041 Grenoble Cedex
}

Le but d'un industriel, constructeur de machines pouvant être soumises aux usures d'érosion par cavitation, est de posséder si possible les matériaux adaptés capables de résoudre ses difficultés.

Nous avons donc sélectionné et testé certains matériaux en partant de matériaux métalliques classiques tels qu'aciers martensito ferritique, dupleix, et en évoluant ensuite vers des alliages type stellite et alliages à faible énergie de faute d'empilement ou, vers des composés organiques. Un de nos principaux soucis était la mise en œuvre facile du matériau sans dégradation du substrat lors du dépôt.

\section{Choix des matériaux}

Les différentes études métallurgiques menées à ce jour sur la tenue des matériaux à la cavitation, indiquent que les propriétés à prendre en compte sont essentiellement: ductilité, écrouissage, limite de rupture et structure à faible énergie de fautes d'empilement.

Les matériaux utilisés dont les caractéristiques sont indiquées dans le tableau ci-dessous récapitulent les matériaux usuels et des matériaux nouveaux dont le choix a été guidé par les propriétés mentionnées ci-dessus.

\begin{tabular}{|l|c|c|c|c|c|c|c|c|c|c|c|c|}
\hline Type & C & Mn & Si & S.10+3 & P.10+3 & G & Ni & Mo & Co & Cu & structure & Hv \\
\hline 13.4 & 0,04 & 0,50 & 0,30 & 15 & 24 & 12,3 & 3,9 & 0,5 & & & c c & 300 \\
\hline 17.4 & 0,05 & 0,55 & 0,32 & 15 & 30 & 16,3 & 4,4 & 0,05 & & & c c & 300 \\
\hline CuAl & $\mathrm{Al}=9,4$ & 1,20 & 0,08 & $\mathrm{Zn} 0,08$ & $\mathrm{Fe} 4,3$ & $\mathrm{Sn} 0,6$ & 4,5 & $\mathrm{~Pb} 0,2$ & & Solde & & 195 \\
\hline St 9080 & 0,4 & 1 & 1 & & & $25 \mathrm{a} 30$ & $1,5 \mathrm{a} 3,5$ & $4,5 \mathrm{a} 6,5$ & Solde & Fe 3 & grade 21 & 330 \\
\hline St 9060 & 1,1 & & & & & 28 & & Fe 2 & Solde & W6 & grade 6 & 430 \\
\hline St IREQ & $0,15 \mathrm{a} 0,25$ & $8 \mathrm{a} 11$ & $2 \mathrm{a} 3$ & & & $15 \mathrm{a} 18$ & & & $8 \mathrm{a} 10$ & & cf c & \\
\hline Fox 26.7N & 0,05 & 4,2 & 0,6 & & & 25,5 & 7,5 & 2 & & N2 0,32 & cf c. ou c c & 290 \\
\hline Phynox & & & & & & 20 & 17 & 7 & 40 & Fe solde & cf c & 200 \\
\hline
\end{tabular}

\section{Study and resistance of some materials to cavitation}

The objective is to select easily processable metallic and organic materials withstanding very well to cavitation. First laboratory results correlating the anticipated metallurgical approach have been obtained. A comparison has been made regarding the behaviour of organic materials and metallic alloys with respect to cavitation. 
Caractéristiques principales des nouveaux matériaux

a) Phynox

- sa structure est austénitique à l'état recuit ;

- son allongement est $\geqslant 65 \%$. Il est ductile ;

- il possède une faible énergie de fautes d'empilement ;

- par écrouissage, il se transforme facilement en phase $\varepsilon$ hc ; la structure obtenue est stable et très fine.

b) Le composé organique Polyuréthane (PU)

— il restitue environ la moitié de l'énergie incidente qu'il reçoit ;

— il possède un fort pouvoir d'allongement (> 200\%);

- sa dureté (60 Sh A) est relativement importante.

c) L'alliage Z5CN13-4 nitruré

- il possède une dureté superficielle importante (1 $200 \mathrm{HV}$ ) sur une profondeur de l'ordre de $2 \mathrm{~mm}$;

- l'alliage Z5CN13-4 nitruré est traité sous vide.

\section{d) Stellites}

- la caractéristique principale est la dureté.

Les matériaux testés ne présentent pas de difficultés de mise en œuvre sauf les stellites qui ont un faible taux d'allongement et l'alliage $\mathrm{Z} 5 \mathrm{CN} 13-4$ nitruré pour lequel le traitement nécessite une enceinte à vide.

Les échantillons sont sous forme:

- massive pour $\mathrm{CuAl}$;

— de dépôt par soudure 13-4; 17-4; St 9080 ; St 9060 ; St

Ireq ; Fox 26-7N ; Phynox ;

- de revêtement $\mathrm{Pu}$;

— nitruré superficiellement.

\section{Essais en laboratoire}

Ces matériaux ont été testés dans trois veines de cavitation de caractéristiques différentes.

a) Boucle à mercure (Institut de Mécanique de Grenoble)

Quatre éprouvettes sont montées sur le cône d'un Venturi avec ogive centrale.

La durée d'essai est de $8 \mathrm{~h}$, avec " rotation " des éprouvettes entre chaque essai pour atteindre $32 \mathrm{~h}$.

Conditions d'essais :

$$
\begin{aligned}
& \mathrm{P} \text { amont }=2,44 \text { bar } \\
& \mathrm{P} \text { aval }=1,56 \text { bar } \\
& \sigma=\frac{\mathrm{P} \text { aval }}{\mathrm{P} \text { amont }}=0,64
\end{aligned}
$$

Vitesse moyenne au col : $4,5 \mathrm{~m} / \mathrm{s}$

Température d'essai : 17 à $20^{\circ} \mathrm{C}$

Compte tenu de la forte densité du mercure, la vitesse de $6 \mathrm{~m} / \mathrm{s}$ correspond à une vitesse en eau de $70 \mathrm{~m} / \mathrm{s}$.

\section{b) Veine venturi (Neyrpic)}

La section d'essai reproduit un venturi avec une ogive centrale réglable pouvant "optimiser " le régime cavitant. Huit éprouvettes sont montées sur les parois du cône extérieur.

Le $\sigma$ d'essai est constant et égal à 0,38 calculé d'après la formule :

$$
\sigma=\frac{\mathrm{P} \text { aval }+\mathrm{P} \text { atmosph }-\mathrm{Tv}}{\mathrm{P} \text { amont }-\mathrm{P} \text { aval }}
$$

Les vitesses variaient de $17 \mathrm{~m} / \mathrm{s}$ (vitesse minimum d'impact sur $\mathrm{Al}$ ) à $46 \mathrm{~m} / \mathrm{s}$ (vitesse maximum possible dans la veine).

$\mathrm{V} \mathrm{m} / \mathrm{s}=\mathrm{Q} \mathrm{m} \mathrm{m}^{3 / \mathrm{s}} / \mathrm{S} \mathrm{m}^{2}, \mathrm{~S}$ étant la section au col de la veine.

Les essais se sont déroulés sur les mêmes éprouvettes soumises successivement aux différents paliers de vitesse avec :

- Pas de $1 \mathrm{~m} / \mathrm{s}$

- Durée de l'essai de 0,5 à $1 \mathrm{~h}$

- Rotation des éprouvettes entre chaque essai pour éliminer les effets de non axisymétrie de l'écoulement.

\section{c) Boucle d'essais caversim (ACB - CERG - Grenoble)}

Cette boucle permet de reproduire, dans une enceinte confinée, l'implosion sur un matériau d'un tourbillon de vapeur.

Les conditions d'essais étaient :

$\begin{array}{ll}\text { - Débit } & : 53,4 \mathrm{l} / \mathrm{s} \\ \text { - } \mathrm{N} \text { obturateur } & : 300 \mathrm{t} / \mathrm{mn} \\ \text { - P aval } & : 1 \mathrm{bar} \text { absolu } \\ \text { - P amont } & : 3,47 \text { bars absolus } \\ \text { - Température de l'eau } & : 12,4^{\circ} \mathrm{C} \\ \text { - Taux } \mathrm{O}_{2} \text { dissous } & : 0,7 \text { à } 0,9 \mathrm{ppm}\end{array}$

La durée de l'essai effectué était de 20 heures. Cette durée de l'essai apparaît insuffisante pour effectuer un classement précis des matériaux métalliques.

\section{Résultats}

\section{a) Campagne d'essais en veine Mercure}

Les conditions de cavitation sont considérées comme peu agressives par rapport à l'essai $\mathrm{c}$.

Les photos (fig. 1) mettent en évidence:

— un très bon comportement de l'acier $\mathrm{Z5CN13-4} \mathrm{nitruré}$ et du PU;

- un comportement similaire du Phynox et de la stellite «IREQ ";

— un bon comportement de la stellite 9060 .

\section{b) Campagne d'essais en Veine Venturi}

Classe les matériaux par rapport à leur comportement à l'usure. Comportement défini par le marquage de l'échan- 


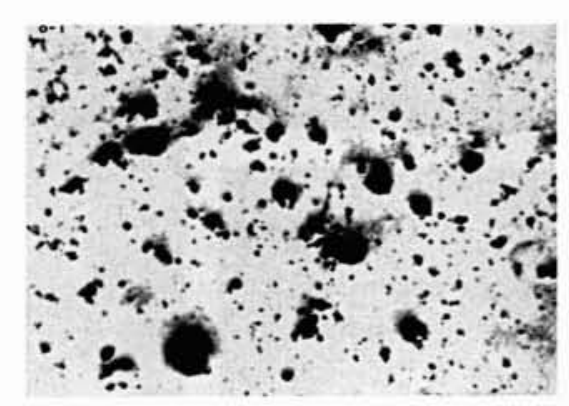

$13 / 4$ NITRURE

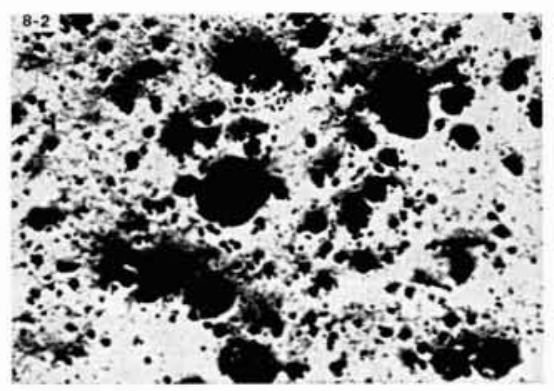

ST N 9060

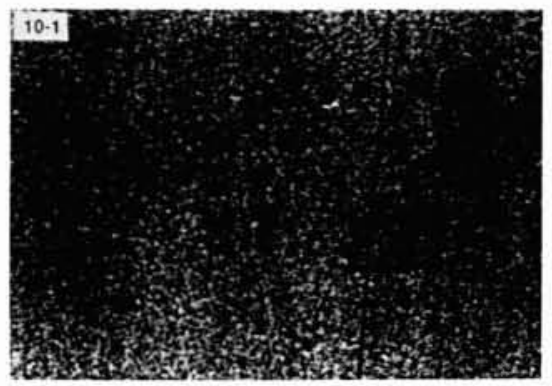

PU AVANT ESSAI

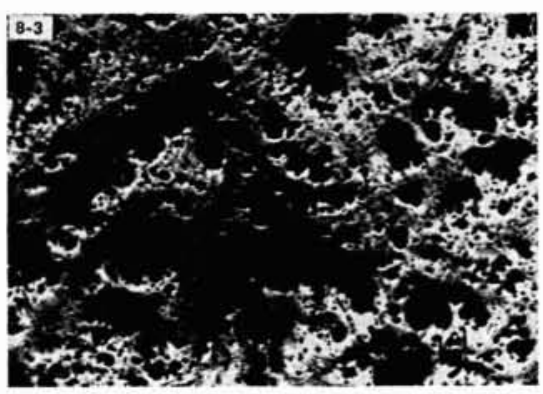

ST IREQ

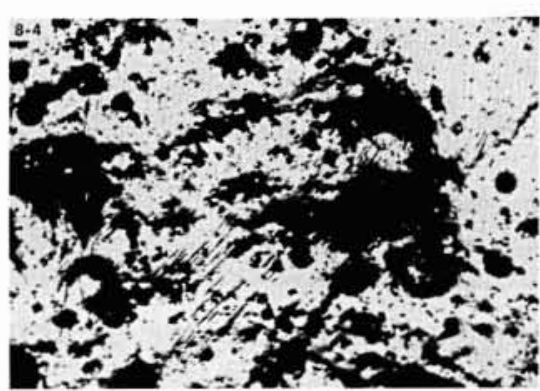

PHYNOX

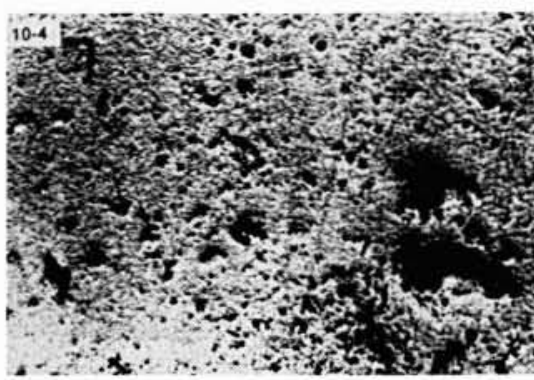

PU APRES ESSAI

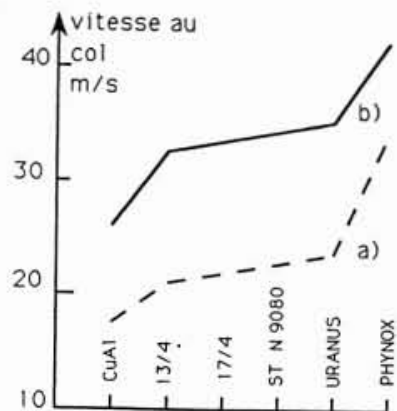

2. Veine Venturi

a) début d'impacts et/ou glissements

b) début des fissures et/ou des arrachements.

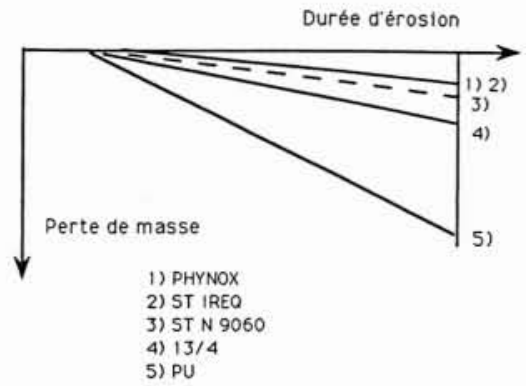

3. Veine Caversim.

1. Résultats des essais après 24 heures d'exposition en veine mercure.

tillon par l'impact d'implosion et le début d'enlèvement de matière vis-à-vis d'une agressivité de cavitation que l'on a caractérisé par sa vitesse (fig. 2). Les $\mathrm{CuAl}$ sont les moins résistants.

Les aciers Z5CN13-4, Z5CN16-4 et la stellite (9080) ont un comportement similaire.

L'examen au microscope métallographique a nettement mis en évidence que la détérioration de la stellite se faisait par déchaussement des grains.

Ces alliages étaient soit à l'état massif soit sous forme de rechargement. Le meilleur comportement a été celui relatif à l'alliage Phynox utilisé sous forme de feuillard.

\section{c) Essais effectués en boucle Caversim}

Les conditions de cavitation sont considérées comme agressives. L'acier $\mathrm{Z} 5 \mathrm{CN} 13-4$ nitruré a un très mauvais comportement et "éclate " (déchaussement au niveau des joints de grains et départ immédiat de matière) au bout d'un temps d'exposition très faible.

Les courbes (fig. 3) montrent que :

- les revêtements PU ne confirment pas leurs très bonnes tenues en agressivité moyenne;

- le phynox, stellite, ont un comportement similaire.

Sur le revêtement Phynox ayant été soumis à la cavitation, des caractérisations supplémentaires ont été effectuées. 


\section{d) Analyse spécifique au Phynox}

Une étude complémentaire a été effectuée sur le revêtement Phynox usé par cavitation comprenant :

Filiation de dureté (HV 500)
à $0,6 \mathrm{~mm}$ du centre d'impact
$=271 \mathrm{Hv}$
à $1 \mathrm{~mm} \mathrm{du}$ bord
$=201 \mathrm{Hv}$
à $1,5 \mathrm{~mm}$ du bord
$\simeq 200 \mathrm{Hv}$

Diffraction de rayons $X$ avec les conditions expérimentales définies par A.H. Graham et J.L. Youngblood (1) (rayonnement $\mathrm{Cr} \mathrm{K} \alpha$ ) et mise en évidence dans la zone endommagée de phase $\varepsilon$ qui correspond à un écrouissage local (fig. 4).

Examen de l'endommagement par étude au $M E B$ (fig. 6) et mise en évidence :

- Présence de cavités dans la zone centrale.

- Début de formation de cavités dans la zone intermédiaire.

- Traces superficielles d'un écrouissage local important conduisant à une initiation de l'endommagement dans la zone périphérique.

Les ruptures sont de type ductile.

L'étude métallurgie physique du revêtement, notamment par microscopie électronique par transmission pour déterminer la taille et le type des enchevêtrements en profondeur dans l'alliage, est en cours.

\section{Essais industriels}

Sur trois sites industriels, des essais comparatifs ont été effectués en rechargeant côte à côte, dans les zones cavitantes, les matériaux à tester (fig. 5) :

a) Brommat

Rechargement d'acier Z5CN16-4 et de stellite 9080.

Après $2800 \mathrm{~h}$ d'exposition, le rapport d'usure entre ces deux matériaux est de 1 à 8 en faveur de la stellite.

Après $8000 \mathrm{~h}$ d'exposition, ce rapport passe de 1 à 2 .

La question qui se pose pour expliquer cet écart, est celle de l'influence éventuelle d'un écrouissage superficiel supérieur de la stellite lors de la remise au profil par meulage ou encore s'agit-il d'une période d'incubation différente?

b) Grand Val

Rechargement de stellites 9060 ; 9080 ; Z5CN16-4 et Fox $26-7 \mathrm{~N}$.

Après $6200 \mathrm{~h}$ d'exposition, les rapports relatifs de tenue entre les matériaux sont :

$\begin{array}{ll}\text { St } 9080 / \text { Z5CN16-4 } & \text { de } 1 \text { à } 3 \\ \text { St } 9080 / \text { FOX } 26-7 N & \text { de } 1 \text { à } 4 \\ \text { St } 9060 / \text { St } 9080 & \text { de } 1 \text { à } 2\end{array}$

c) Beaumont Monteux

Rechargement Z5CN16-4; Phynox et Fox 26-7N

Les rapports d'usure entre ces matériaux sont:

Z5CN16-4/FOX 26-7N
Phynox/Z5CN16-4

614

\section{Comparaison des résultats}

Les essais industriels semblent mettre en évidence une bonne tenue de la stellite 9060 . Tenue légèrement supérieure à la stellite 9080 qui est comparable à la stellite "IREQ".

La tenue du Phynox est comparable à celle de la stellite 9080 mais présente le très gros avantage d'une mise en œuvre très facile et ne présente pas lors du dépôt, des problèmes de fissuration.

Les essais en boucle à mercure semblent confirmer ces résultats.

Toutefois, les résultats de " faible agressivité » en boucle mercure ne sont pas confirmés pour :

- le Z5CN13-4 durci superficiellement par nitruration qui lors d'une cavitation plus agressive en boucle Caversim se détache facilement par déchaussement immédiat des grains ;

- le PU n'a pas ses résultats confirmés par un essai dans une autre boucle. Il est nécessaire de vérifier la tenue de ce dernier en conditions d'usure industrielle.



4. Diagramme de rayons $X$ sur échantillon Phynox.

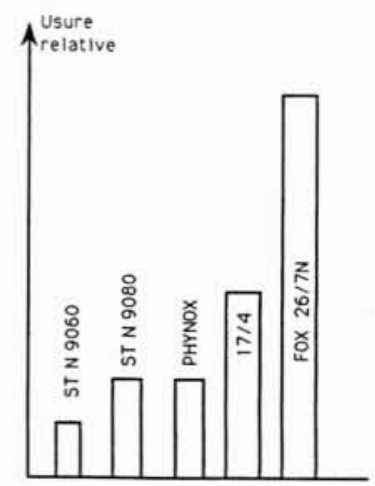

5. Résultats industriels.

\section{Remerciements}

Nous remercions la société Imphy pour l'aide qu'elle nous

a apportée lors de ce travail. de l'ordre de 2 de l'ordre de 2 

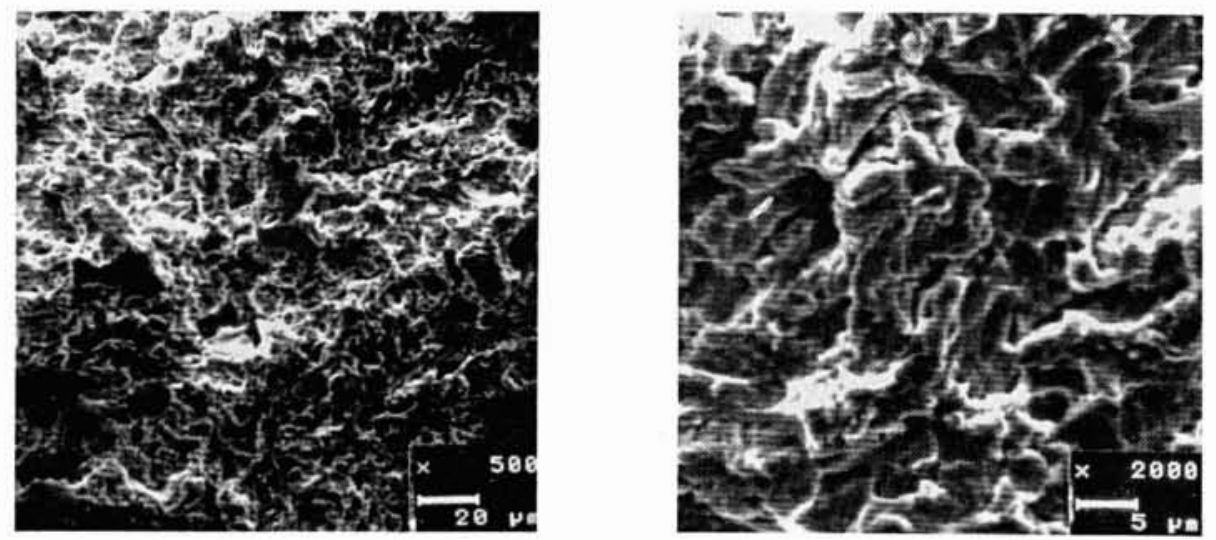

ZONE CENTRALE
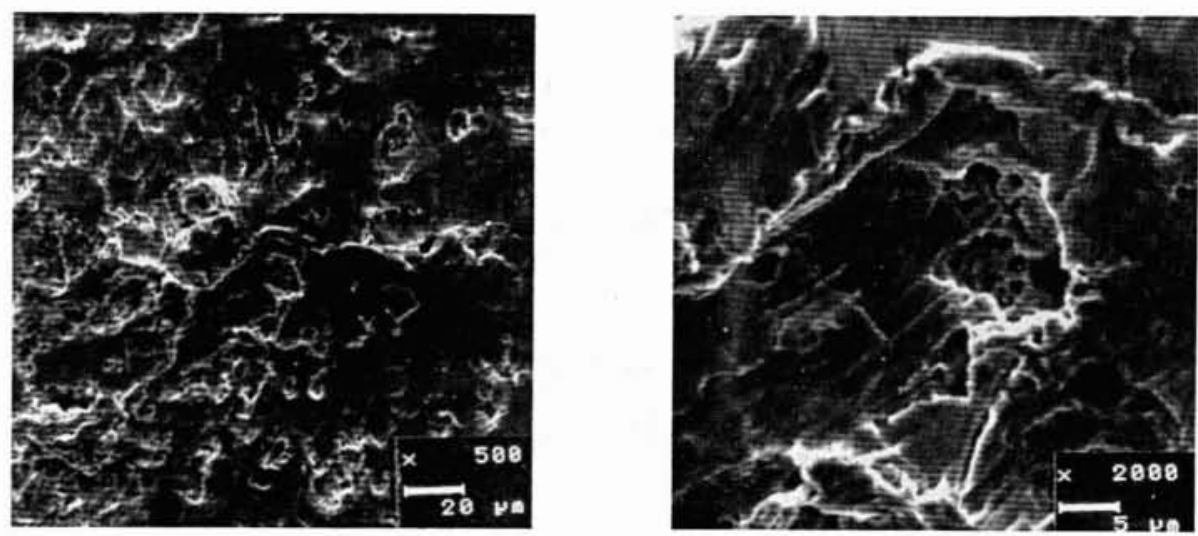

ZONE INTERMEDIAIRE
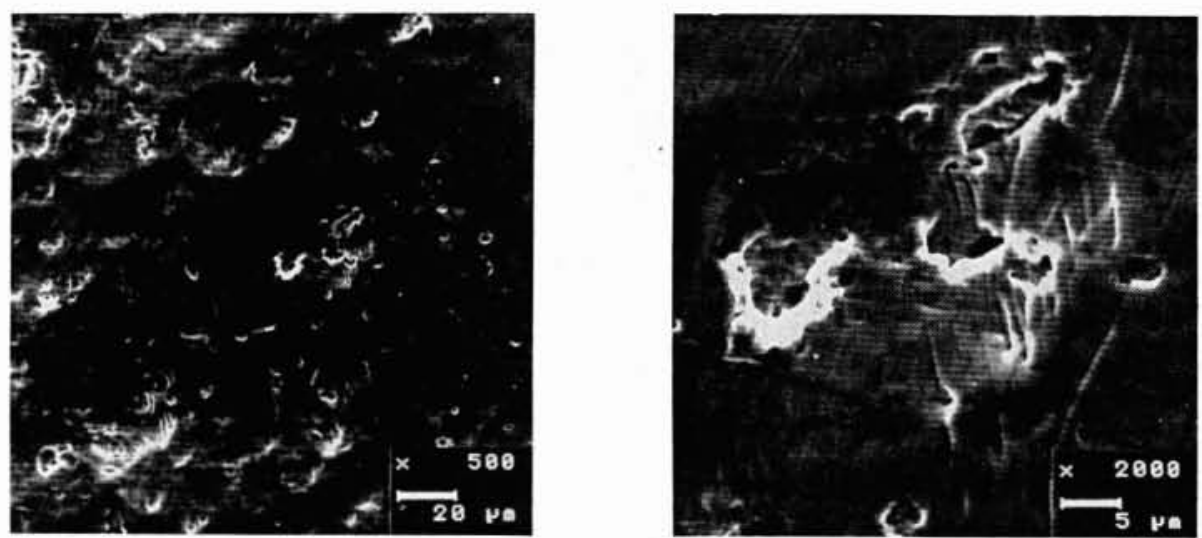

ZONE PERIPHERIQUE

6. Images au M.E.B. du Phynox après cavitation en veine Caversim. 




